Laszczak Katarzyna, Moqbil Sara, Niedobylski Sylwiusz, Warchoł Konrad. Cardiovascular comorbidities in acromegaly. Journal of Education, Health and Sport. 2021;11(8):283-290. eISSN 2391-8306. DOI http://dx.doi.org/10.12775/JEHS.2021.11.08.030 https://apcz.umk.pl/czasopisma/index.php/JEHS/article/view/JEHS.2021.11.08.030

https://zenodo.org/record/5231536

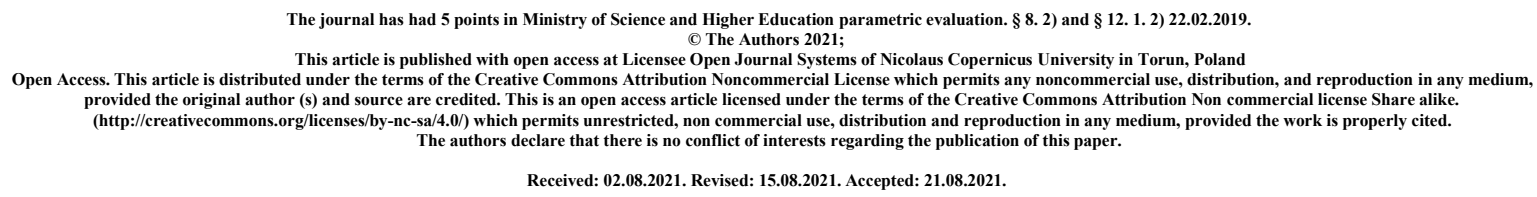

\title{
Cardiovascular comorbidities in acromegaly
}

\author{
Katarzyna Laszczak, Sara Moqbil, Sylwiusz Niedobylski, Konrad Warchoł \\ Katarzyna Laszczak (1); 0000-0002-5084-0273 \\ Sara Moqbil (1); 0000-0003-1230-1444 \\ Sylwiusz Niedobyski (1); 0000-0001-7266-623X \\ Konrad Warchol (1); 0000-0001-9467-680X
}

(1) Student Scientific Society at the Department of Endocrinology, Independent Public Clinical Hospital No. 4 in Lublin, ul. Jaczewskiego 8 20-954 Lublin

\begin{abstract}
Introduction and purpose: Acromegaly is a rare disease which occurs with the frequency of 0.2-1.1 cases per 100000 patients per year. The main cause is the excess level of growth hormone $(\mathrm{GH})$ which stimulates the liver to the insulin-like growth factor type 1 (IGF-1) secretion. IGF-1 leads to the tissues overgrowth. In addition, acromegalic patients suffer from many comorbidities, such as: cardiovascular, endocrinological, neoplastic and musculoskeletal complications. The main aim of this review is the update of the latest information concerning cardiovascular comorbidities in patients with acromegaly.

State of knowledge: Studies revealed that $80 \%$ of acromegalic patients will develop cardiovascular comorbidities. Complications such as: hypertension, cardiomyopathy, arrhythmia, heart valve disease, atherosclerosis and coronary heart disease as well as myocardial infarction were the most widely described. They are responsible for $44 \%$ of deaths in the first decade of the acromegaly and $23 \%$ in the second one.

Conclusions: Because of the delayed diagnosis of acromegaly in most patients, most comorbidities are discovered in advanced stages leading to the decrease in patients' life span. Adequate knowledge about potential complications is necessary to increase patients' quality of lives.
\end{abstract}

Key words: acromegaly, cardiovascular comorbidities, hypertension, cardiomyopathy, arrhythmia, heart valve disease 


\section{Introduction:}

Acromegaly is an underdiagnosed endocrine disease. Its prevalence is estimated for $0.2-1.1$ cases per 100000 patients per year [1]. In 95\% cases acromegaly is caused by macroadenoma of the pituitary gland, which secretes excessive amounts of growth hormone (GH) levels [2]. It stimulates the liver to produce insulin-like growth factor type 1 (IGF-1), known as somatomedin $\mathrm{C}$. This factor has a huge impact on tissues and leads to their overgrowth [3]. Enlarged organs do not function properly which is the cause of many comorbidities, such as: arthritis, prognathism, glucose intolerance, cardiovascular, neoplastic and musculoskeletal complications [4], [5].

Studies showed that in most patients acromegaly is diagnosed in the 40 years of age. In majority cases this disease is discovered 10 years after the occurrence of first symptoms. It means that in many patients comorbidities are detected in the advanced state [6]. The most common complications are cardiovascular and pulmonary ones. They lead to the decrease of patients' life span by $30 \%$ [4].

Studies revealed that $80 \%$ of acromegalic patients will develop cardiovascular comorbidities [7]. Subsequently, they are responsible for $44 \%$ of deaths in the first decade of the disease and $23 \%$ in the second one [8]. Among cardiovascular complications it is necessary to mention: hypertension, cardiomyopathy, arrhythmia, heart valve disease, atherosclerosis and coronary heart disease, myocardial infarction as well as congestive heart failure[9]-[11].

The main aim of this review is the update of the latest information concerning cardiovascular comorbidities in patients with acromegaly. We are going to reveal their epidemiology, as well as pathogenesis.

\section{Hypertension}

Arterial hypertension is one of the most frequent cardiovascular complications of acromegaly [12]. The disease greatly contributes to the excess mortality in acromegalic patients [13], and increases its hospitalisation rate as well as general healthcare costs [14]. The prevalence of hypertension in acromegalic patients ranges from 11\% (Denmark, [15]), up to 54,7\% (New Zealand, [13]). The mean frequency is estimated to be 33,6\% [16]. The differences may arise from different diagnostic criteria for hypertension, as well as various population-related risk factors.

Multifactorial origin is the most widely accepted mechanism for hypertension pathogenesis. The receptor for GH was proven to be expressed in adrenal cortex, therefore possibly being connected to the aldosterone secretion [17]. Additionally the in vivo studies showed antinatriuretic properties of $\mathrm{GH}$ in rats displayed in poor response of furosemide, which confirmed the mechanism of action of the hormone - GH seems to stimulate epithelial sodium channels in distal nephron tubules [18]. GH may also influence arterial pressure through the renin-angiotensin-aldosterone system. Previously mentioned $\mathrm{GH}$ receptors expressed on adrenal cortex may be the reason of a correlation of $\mathrm{GH}$ and aldosterone values in acromegalic mice samples, with the normalisation of aldosterone concentrations after decreasing GH secretion through adenoma surgery. The renin-angiotensin-aldosterone system upregulation was independent of renin or IGF-1 expression [19]. Other studies show the impact of IGF-1 increased secretion on hypertension risk, although through other mechanisms IGF-1 seems to reduce secretion of atrial natriuretic peptide (ANP), which leads to the sodium accumulation. Acromegalic patients have significantly lower response in ANP levels after saline venous infusion, in comparison to the control group, in which the notable increase in the peptide levels was recorded. Basal levels of ANP were similar in both groups in this study [20]. The influence of IGF-1 on this pathology was found in 2000 by Moller et al. [21]. Other probable factor associated with acromegaly is hyperinsulinemia, which often occurs in that disease along with insulin resistance. The levels of insulin in oral glucose tolerance in 
acromegalic patients with hypertension seem to be higher than in normotensive patients [22], [23]. Another mechanisms include increase of vascular resistances in peripheral blood vessels due to $\mathrm{GH} / \mathrm{IGF}-1$-mediated endothelial dysfunction with underlying nitrous oxide activity change [24], or sympathetic activation [25]. Another cause of blood pressure increase might be sleep apnea, a common comorbidity of acromegaly.

\section{Cardiomyopathy}

Acromegalic cardiomyopathy may occur early during disease, with a prevalence of approximately $70 \%$ [26], and even up to $90 \%$ in hypertensive patients [27]. It can coexist with other cardiovascular risk factors, such as hypertension and type 2 diabetes, which could accelerate the hypertrophy of cardiac muscle. Subsequently, it also may be found in patients without other cardiac comorbidities, due to the direct effect on cardiac muscle cells [28].

GH's and IGF-1's proliferative effect promotes the synthesis of sarcomeric proteins, collagen deposition, lymphomononuclear infiltration, dysregulation in cellular water balance, while also binding with its specific receptor, altering muscle contractility [29][30][31]. All of the above factors contribute to architectural change - biventricular, concentric overgrowth of heart muscle; fiber hypertrophy; cell degeneration and fibrosis, which leads to systolic and diastolic dysfunction, low cardiac output and, eventually, heart failure [32].

We can differentiate cardiomyopathy into three stages. First, with concentric hypertrophy and hyperkinetic left ventricle, leading to increased systolic output and increased contractility. In that stage, the patient can observe increased heart rate. Second, with progressing hypertrophy and fibrosis, diastolic filling and ejection fraction are reduced. This is the stage that most of the patients are diagnosed with, due to decreased exercise capacity. Third, end-stage cardiomyopathy, with both systolic and diastolic dysfunction, usually found in untreated acromegaly, ultimately leads to congestive heart failure [32][33][34][35]. Bihan et al. estimated that less than $3 \%$ of patients with acromegaly could develop congestive heart failure, but the final prognosis for those patients is rather poor [36]. Potential risk factors are advancing age, disease duration, activity, management, and diabetes [32][37].

In diagnosis, the main evaluation tool is ultrasound, but not being sensitive and specific, cardiac magnetic resonance (CMR) is considered to be a gold-standard method [26][38].

It is suggested that the key element to decelerate the progression of changes in the heart is to reduce exposure to elevated levels of GH and IGF-1 [39]. Merola et al. [40] study comprising 11 patients with active acromegaly, showed that 6-month octreotide treatment improved cardiac structure and function.

\section{Arrhythmia}

Heart rhythm abnormalities are frequent findings in acromegalic patients. The most common are paroxysmal atrial fibrillation (AF), paroxysmal supraventricular tachycardia (SVT), sick sinus syndrome, ventricular tachycardia (VT), ventricular ectopic beats, and bundle branch blocks, usually recorded at peak exercise [41]. Beat-to-beat QT variability and increased prevalence of late potentials are considered to be risk factors for arrhythmia and could be used as an indicator for in-depth diagnosis [42][43]. Left ventricular dyssynchrony, being the loss of the simultaneous peak contraction of corresponding cardiac segments, was also linked with disease activity and levels of GH and IGF-1 [44].

Fibrosis and derangement of muscle fibers in the heart are thought to be one of the reasons for arrhythmia formation, as slowed and dissociated potentials are conducted through deformed tissue [45]. Chatterjee et al. [46], in their meta-analysis, consisting of 27141 patients with left ventricular hypertrophy, not suffering from acromegaly, confirmed that change in the structure of the heart makes one prone to arrhythmias. For instance, SVT was present in $11.1 \%$ of patients with hypertrophy, compared with $1.1 \%$ among patients without hypertrophy, and for VT risk for affected patients was 2.8-fold greater than not affected with hypertrophy. 
Control of GH and IGF-1 levels seems to be crucial for the prevention of cardiac arrhythmias. Warszawski et al. [47] conducted a study on 36 patients with acromegaly, previously not treated with somatostatin analogs. Although not entirely confident, researchers assume, thanks to slowed or entirely halted changes in cardiac muscle, the frequency of arrhythmic incidents may be lowered.

\section{Heart valve disease}

Studies showed that among the general population, in a wide-age group heart valve disease occurs in $0.7-40 \%$ people. In comparison over $75 \%$ of acromegalic patients suffer from valvular heart [48]. The most frequently affected are mitral and/or aortic valves [49].

The main causes of valve impairment of the function are their thickening, fibrosis and calcification [48]. GH leads to dysregulation of metalloproteinase and proteoglycan secretion. There is an increase in mucopolysaccharide accumulation in the valves and collagen reduction [50]. Because of the increased number of GH and IGF-1, patients with acromegaly have higher amounts of pro-inflammatory cytokines, which raise the metalloproteinase expression [51]. These enzymes degenerate collagen, proteoglycans as well as elastin, of which valves consist of. That lead to the changes in the valve construction and in consequence their dysfunction [52]. In $20 \%$ of patients, valves modifications are mild or moderate [4].

The duration of acromegaly has direct correlation with valve degeneration. The main cause of this phenomenon is high exposition to GH. The progression of heart valve disease is increased by cardiovascular risk factors, such as widened aortic root diameter [53].

Detecting heart valve disease in acromegalic patients is highly important as it may lead to the ventricular hypertrophy and arrhythmias [50].

\section{Atherosclerosis and coronary heart disease}

Increased plasma level of lipoprotein(a) (Lpa) is a risk factor of cardiovascular diseases. The levels of $\mathrm{Lp}(\mathrm{a})$ and $\mathrm{GH}$ are correlated in patients with acromegaly. There are studies that prove that active acromegalics are at higher risk for early atherosclerotic lesions due to high prevalence of risk factors. It has been demonstrated that active acromegalics have higher carotid artery intima-media-thickness (IMT) and their brachial artery flow-mediated dilation (FMD) levels are significantly lower than in matched control subjects [54]. Abnormal GH and IGF-1 secretion leads to the development of acromegalic cardiomyopathy. The correlation between IGF-1 levels and development of atherosclerosis and coronary heart disease has been proven. The study on 95 patients who had previously had myocardial infarctions and 92 probands showed that patients with coronary heart disease (CHD) have significantly higher serum concentrations of IGF-1 than age- and BMI-matched controls [55]. The Framingham risk score (FRS) - a gender-specific algorithm used to estimate the 10-year cardiovascular risk of an individual is higher in acromegalic patients compared with matched control subjects $(13.6 \pm 7.9$ vs. $10.6 \pm 7.7 \%)$ [56]. In addition, calcium store is another highly sensitive and specific indicator to determine the risk of developing coronary artery disease. An integrated assessment of FRS and Agatston score (AS) on 39 patients showed that $41 \%$ of acromegalics were at risk for coronary atherosclerosis, and coronary calcification was evident in about half of them [12]. Studies in smaller cohorts admittedly have not shown an association of coronary artery disease with acromegaly activity, however, they have indicated the presence of acromegaly-related systemic complications, like hyperlipidemia and diabetes, that significantly increase the risk of future coronary artery disease [57], [58].

\section{Myocardial infarction}

The increased morbidity and mortality in acromegaly is mainly attributed to cardiovascular diseases that may subsequently increase the risk of a myocardial infarction. Among the 3173 acromegalics studied, the incidence of myocardial infarction at diagnosis was $3.0 \%$ [59]. Another study of 27 acromegaly patients undergoing autopsy found that $15 \%$ of them had a history of a heart attack [60]. Late potentials in the signal-averaged electrocardiogram 
(SAECG) are often observed in patients who have had a history of myocardial infarction. A study involving 48 patients with acromegaly showed that late potentials were detected in $56 \%$ of patients with active acromegaly compared to $6 \%$ in the cured/well-controlled acromegaly group, the incidence of late potentials was significantly higher in patients with acromegaly $(23 \%)$ than in the control group (0\%) [61]. Another factor underlying the pathophysiology of heart failure and myocardial infarction is obstructive sleep apnea (OSA) [62], [63] which is often associated with acromegaly. In cross-sectional study, despite clear evidence that patients with acromegaly are at risk for coronary atherosclerosis and coronary calcification is common, the incidence of myocardial infarction was not significantly higher compared with the general population [64]. A recent study indicates that among patients with acromegaly treated in specialized centers, the incidence of heart attacks and strokes does not appear to differ from the general population [65].

\section{Conclusions}

Cardiovascular comorbidities occur in most cases of acromegalic patients. They increase the mortality rate and decrease patients' quality of lives. The most known cardiac complications are: hypertension, cardiomyopathy, arrhythmia, heart valve disease, atherosclerosis and coronary heart disease, myocardial infarction as well as congestive heart failure. Because of the major problem which is patients death due to cardiovascular comorbidities further investigations on that area are necessary.

\section{References:}

1. A. Lavrentaki, A. Paluzzi, J. A. H. Wass, and N. Karavitaki, 'Epidemiology of acromegaly: review of population studies.', Pituitary. 2017;20(1):4-9. DOI: 10.1007/s11102016-0754-x.

2. L. Vilar, C. F. Vilar, R. Lyra, R. Lyra, and L. A. Naves, 'Acromegaly: clinical features at diagnosis', Pituitary. 2017;20(1):22-32. DOI: 10.1007/s11102-016-0772-8.

3. D. S. Greco, 'Feline acromegaly.', Top Companion Anim Med. 2012;27(1):31-35. DOI: 10.1053/j.tcam.2012.05.004.

4. S. Melmed, 'Acromegaly pathogenesis and treatment.', The Journal of clinical investigation. 2009;119(11):3189-3202. DOI: 10.1172/JCI39375.

5. M. R. Ambrosio et al., 'Acromegaly in the elderly patients.', Endocrine. 2020;68(1):1631 DOI: $10.1007 / \mathrm{s} 12020-020-02206-7$.

6. P. Chanson and S. Salenave, 'Acromegaly.', Orphanet journal of rare diseases. 2008;3:17. DOI: 10.1186/1750-1172-3-17.

7. I. M. Holdaway, R. C. Rajasoorya, and G. D. Gamble, 'Factors influencing mortality in acromegaly.', J Clin Endocrinol Metab. 2004;89(2):667-674. DOI: 10.1210/jc.2003-031199.

8. M. Mercado et al., 'Successful mortality reduction and control of comorbidities in patients with acromegaly followed at a highly specialized multidisciplinary clinic.', $J$ Clin Endocrinol Metab. 2014;99(12):4438-4446. DOI: 10.1210/jc.2014-2670.

9. S. Puglisi and M. Terzolo, 'Hypertension and Acromegaly.', Endocrinol Metab Clin North Am. 2019;48(4):779-793 DOI: 10.1016/j.ecl.2019.08.008.

10. M. D. Sharma, A. V. Nguyen, S. Brown, and R. J. Robbins, 'Cardiovascular Disease in Acromegaly.', Methodist Debakey Cardiovasc J. 2017;13(2):64-67. DOI: 10.14797/mdcj-13$2-64$.

11. R. N. Clayton, 'Cardiovascular function in acromegaly.', Endocr Rev. 2003;24(3):272277. DOI: 10.1210/er.2003-0009.

12. M. Ragonese et al., 'Cardiovascular events in acromegaly: distinct role of Agatston and Framingham score in the 5-year prediction', Endocrine. 2014;47(1):206-212. DOI: 10.1007/s 12020-013-0115-8. 
13. I. M. Holdaway, R. C. Rajasoorya, and G. D. Gamble, 'Factors influencing mortality in acromegaly', J Clin Endocrinol Metab. 2004;89(2):667-674. DOI: 10.1210/jc.2003-031199.

14. M. S. Broder, M. P. Neary, E. Chang, D. Cherepanov, and L. Katznelson, 'Treatments, complications, and healthcare utilization associated with acromegaly: a study in two large United States databases', Pituitary. 2014;17(4):333-341 DOI: 10.1007/s11102-013-0506-0.

15. J. Dal et al., 'Acromegaly incidence, prevalence, complications and long-term prognosis: a nationwide cohort study', Eur J Endocrinol. 2016;175(3):181-190. DOI: 10.1530/EJE-160117.

16. S. Puglisi and M. Terzolo, 'Hypertension and Acromegaly', Endocrinology and Metabolism Clinics of North America. 2019;48(4):779-793. DOI: 10.1016/j.ecl.2019.08.008.

17. C. J. Lin, B. B. Mendonca, A. M. Lucon, I. C. Guazzelli, W. Nicolau, and S. M. Villares, 'Growth hormone receptor messenger ribonucleic acid in normal and pathologic human adrenocortical tissues--an analysis by quantitative polymerase chain reaction technique', $J$ Clin Endocrinol Metab. 1997;82(8):2671-2676. DOI: 10.1210/jcem.82.8.4159.

18. P. Kamenicky et al., 'Epithelial sodium channel is a key mediator of growth hormoneinduced sodium retention in acromegaly', Endocrinology. 2008;149(7):3294-3305. DOI: 10.1210/en.2008-0143.

19. M. Bielohuby et al., 'Chronic growth hormone excess is associated with increased aldosterone: a study in patients with acromegaly and in growth hormone transgenic mice', Exp Biol Med (Maywood). 2009;234(8):1002-1009. DOI: 10.3181/0901-RM-34.

20. J. A. McKnight et al., 'Basal and saline-stimulated levels of plasma atrial natriuretic factor in acromegaly', Clin Endocrinol (Oxf). 1989;31(4):431-438. DOI: 10.1111/j.13652265.1989.tb01267.x.

21. J. Møller, J. O. Jørgensen, J. Marqversen, E. Frandsen, and J. S. Christiansen, 'Insulinlike growth factor I administration induces fluid and sodium retention in healthy adults: possible involvement of renin and atrial natriuretic factor', Clin Endocrinol (Oxf). 2000;52(2):181-186. DOI: 10.1046/j.1365-2265.2000.00931.x.

22. J. Słowińska-Srzednicka, S. Zgliczyński, P. Soszyński, W. Zgliczyński, and W. Jeske, 'High blood pressure and hyperinsulinaemia in acromegaly and in obesity', Clin Exp Hypertens A. 1989;11(3):407-425. DOI: 10.3109/10641968909035351.

23. T. Ikeda et al., 'Correlation between blood pressure and plasma insulin in acromegaly', $J$ Intern Med. 1993;234(1):61-63. DOI: 10.1111/j.1365-2796.1993.tb00705.x.

24. V. Ronconi et al., 'Reduced nitric oxide levels in acromegaly: cardiovascular implications', Blood Press. 2005;14(4):227-232. DOI: 10.1080/08037050510034293.

25. M. Bondanelli, M. R. Ambrosio, and E. C. degli Uberti, 'Pathogenesis and prevalence of hypertension in acromegaly', Pituitary. 2001;4(4):239-249. DOI: 10.1023/a:1020798430884.

26. F. Bogazzi et al., 'High prevalence of cardiac hypertophy without detectable signs of fibrosis in patients with untreated active acromegaly: an in vivo study using magnetic resonance imaging', Clinical Endocrinology. 2008;68(3):361-368. DOI: 10.1111/J.13652265.2007.03047.X.

27. L. JT, 'Pathology of the heart in acromegaly: anatomic findings in 27 autopsied patients', American heart journal. 1980;100(1):41-52. DOI: 10.1016/0002-8703(80)90277-X.

28. A. Colao, P. Marzullo, C. Di Somma, and G. Lombardi, 'Growth hormone and the heart', Clinical Endocrinology. 2001;54(2):137-154. DOI: 10.1046/J.1365-2265.2001.01218.X.

29. N. S. Freestone, S. Ribaric, and W. T. Mason, 'The effect of insulin-like growth factor-1 on adult rat cardiac contractility', Molecular and Cellular Biochemistry 1996 163:1. 1996;163(1):223-229. DOI: 10.1007/BF00408662.

30. F. ML et al., 'Myocardial hypertrophy in transgenic mice overexpressing the bovine growth hormone (bGH) gene', Journal of internal medicine. 2000;247(5):546-552. DOI: 10.1046/J.1365-2796.2000.00651.X. 
31. H. Gouya et al., 'Rapidly Reversible Myocardial Edema in Patients with Acromegaly: Assessment with Ultrafast T2 Mapping in a Single-Breath-Hold MRI Sequence', http://dx.doi.org/10.2214/AJR.07.2031. 2012;190(6):1576-1582. DOI: 10.2214/AJR.07.2031. 32. G. MD, V. N, Y. S, and F. WH, 'Acromegalic Cardiomyopathy: An Overview of Risk Factors, Clinical Manifestations, and Therapeutic Options', Cardiology in review. 2018;26(6):307-311 DOI: 10.1097/CRD.0000000000000215.

33. L. Saccà, R. Napoli, and A. Cittadini, 'Growth hormone, acromegaly, and heart failure: an intricate triangulation', Clinical Endocrinology. 2003;59(6):660-671. DOI: 10.1046/J.1365-2265.2003.01780.X.

34. R.-L. AM and M. M, 'Cardiovascular comorbidities in acromegaly: an update on their diagnosis and management', Endocrine. 2017;55(2):346-359. DOI: 10.1007/S12020-0161191-3.

35. A. B et al., 'Evaluation of left ventricular diastolic function according to new criteria and determinants in acromegaly', International heart journal. 2012;53(5):299-305, 2012. DOI: 10.1536/IHJ.53.299.

36. B. H et al., 'Long-term outcome of patients with acromegaly and congestive heart failure', The Journal of clinical endocrinology and metabolism. 2004;89(11):5308-5313. DOI: 10.1210/JC.2004-0821.

37. I. J, A. M, K. K, and C. A, 'GH and the cardiovascular system: an update on a topic at heart', Endocrine. 2015;48(1):25-35. DOI: 10.1007/S12020-014-0327-6.

38. P. M, D. F, V. R, and M. P, 'Acromegaly and ultrasound: how, when and why?', Journal of endocrinological investigation. 2020;43(3):279-287. DOI: 10.1007/S40618-019-01111-9.

39. A. Colao, D. Ferone, P. Marzullo, and G. Lombardi, 'Systemic Complications of Acromegaly: Epidemiology, Pathogenesis, and Management', Endocrine Reviews. 2004;25(1):102-152. DOI: 10.1210/ER.2002-0022.

40. M. B et al., 'Chronic treatment with the somatostatin analog octreotide improves cardiac abnormalities in acromegaly', The Journal of clinical endocrinology and metabolism. 1993;77(3):790-793. DOI: 10.1210/JCEM.77.3.8370700.

41. K. G et al., 'Arrhythmia profile in acromegaly', European heart journal. 1992;13(1):5156. DOI: 10.1093/OXFORDJOURNALS.EURHEARTJ.A060047.

42. O. A et al., 'Increased Short-Term Beat-To-Beat Variability of QT Interval in Patients with Acromegaly', PloS one. 2015;10(4). DOI: 10.1371/JOURNAL.PONE.0125639.

43. M. P et al., 'Late potentials and ventricular arrhythmias in acromegaly', International journal of cardiology. 2005;104(2):197-203. DOI: 10.1016/J.IJCARD.2004.12.010.

44. K. A et al., 'Left ventricular synchronicity is impaired in patients with active acromegaly', Endocrine. 2013;44(1):200-206. DOI: 10.1007/S12020-012-9859-9.

45. de J. S, van V. TA, van R. HV, and de B. JM, 'Fibrosis and cardiac arrhythmias', Journal of cardiovascular pharmacology. 2011;57(6):630-638. DOI: 10.1097/FJC.0B013E318207A35F.

46. C. S et al., 'Meta-analysis of left ventricular hypertrophy and sustained arrhythmias', The American journal of cardiology. 2014;114(7):1049-1052. DOI: 10.1016/J.AMJCARD.2014.07.015.

47. W. L et al., 'Low frequency of cardniac arrhythmias and lack of structural heart disease in medically-naïve acromegaly patients: a prospective study at baseline and after 1 year of somatostatin analogs treatment', Pituitary. 2016;19(6):582-589. DOI: 10.1007/S11102-0160749-7.

48. A. Colao et al., 'High prevalence of cardiac valve disease in acromegaly: an observational, analytical, case-control study.', J Clin Endocrinol Metab. 2003;88(7):31963201. DOI: $10.1210 /$ jc.2002-021099. 
49. A. M. Ramos-Leví and M. Marazuela, 'Bringing Cardiovascular Comorbidities in Acromegaly to an Update. How Should We Diagnose and Manage Them?', Front Endocrinol (Lausanne). 2019;10:120, 2019. DOI: 10.3389/fendo.2019.00120.

50. A. M. Ramos-Leví and M. Marazuela, 'Cardiovascular comorbidities in acromegaly: an update on their diagnosis and management.', Endocrine. 2017;55(2):346-359. DOI: 10.1007/s12020-016-1191-3.

51. P. Pereira, F. Duarte, R. Lamas, and R. Vaz, 'Idiopathic spinal cord herniation: case report and literature review.', Acta Neurochir (Wien). 2001;143(4):401-406. DOI: $10.1007 / \mathrm{s} 007010170096$.

52. H. Yang, H. Tan, H. Huang, and J. Li, 'Advances in Research on the Cardiovascular Complications of Acromegaly.', Front Oncol. 2021;11:640999. DOI: 10.3389/fonc.2021.640999.

53. A. A. van der Klaauw et al., 'Uncontrolled acromegaly is associated with progressive mitral valvular regurgitation.', Growth Horm IGF Res. 2006;16(2):101-107. DOI: 10.1016/j.ghir.2006.02.002.

54. I. Kartal et al., 'Investigation of early atherosclerotic changes in acromegalic patients.', Int J Clin Pract. 2010;64(1):39-44. DOI: 10.1111/j.1742-1241.2008.01750.x.

55. F. Fischer et al., 'Associations of insulin-like growth factors, insulin-like growth factor binding proteins and acid-labile subunit with coronary heart disease.', Clin Endocrinol (Oxf). 2004;61(5):595-602. DOI: 10.1111/j.1365-2265.2004.02136.x.

56. C. Berg et al., 'Cardiovascular risk factors in patients with uncontrolled and long-term acromegaly: comparison with matched data from the general population and the effect of disease control.', J Clin Endocrinol Metab. 2010;95(8):3648-3656. DOI: 10.1210/jc.20092570 .

57. F. Bogazzi et al., 'Risk factors for development of coronary heart disease in patients with acromegaly: a five-year prospective study.', $J$ Clin Endocrinol Metab. 2007;92(11):4271-4277. DOI: 10.1210/jc.2007-1213.

58. H. Akutsu et al., 'Acromegaly per se does not increase the risk for coronary artery disease.', Eur J Endocrinol. 2010;162(5):879-886. DOI: 10.1530/EJE-09-0945.

59. P. Petrossians et al., 'Acromegaly at diagnosis in 3173 patients from the Liège Acromegaly Survey (LAS) Database', Endocr Relat Cancer. 2017;24(10):505-518. DOI: 10.1530/ERC-17-0253.

60. C. COURVILLE and V. R. MASON, 'THE HEART IN ACROMEGALY', Archives of Internal Medicine. 1938;61(5):704-713. DOI: 10.1001/archinte.1938.00180100014002.

61. B. L. Herrmann et al., 'Occurrence of ventricular late potentials in patients with active acromegaly.', Clin Endocrinol (Oxf). 2001;55(2):201-207. DOI: 10.1046/j.13652265.2001.01319.x.

62. T. Kendzerska, A. S. Gershon, G. Hawker, R. S. Leung, and G. Tomlinson, 'Obstructive sleep apnea and risk of cardiovascular events and all-cause mortality: a decade-long historical cohort study.', PLoS Med. 2014;11(2):1001599. DOI: 10.1371/journal.pmed.1001599.

63. H. Nakashima et al., 'Obstructive sleep apnoea increases the incidence of morning peak of onset in acute myocardial infarction.', Eur Heart J Acute Cardiovasc Care. 2013;2(2):153158. DOI: $10.1177 / 2048872613478557$.

64. S. Cannavo et al., 'Acromegaly and coronary disease: an integrated evaluation of conventional coronary risk factors and coronary calcifications detected by computed tomography.', J Clin Endocrinol Metab. 2006;91(10):3766-3772. DOI: 10.1210/jc.2005-2857. 65. C. Schöfl et al., 'Incidence of myocardial infarction and stroke in acromegaly patients: results from the German Acromegaly Registry.', Pituitary. 2017;20(6):635-642. DOI: 10.1007/s11102-017-0827-5. 